Disclosure of Interests: None declared

DOI: 10.1136/annrheumdis-2019-eular.3949

\section{FRI0225 1 THE EULAR/ACR 2018 AND SLICC 2012 HAVE INCREASED SENSITIVITY AGAINST THE ACR 1997 CLASSIFICATION CRITERIA AND CLASSIFY NON- OVERLAPPING GROUPS OF SLE PATIENTS: SIMULTANEOUS APPLICATION ASSURES THE GREATEST CAPTURE OF PATIENTS IN CLINICAL PRACTICE}

Christina Adamichou ${ }^{1}$, Dionisis Nikolopoulos ${ }^{2}$, Irini Genitsaridi ${ }^{3}$, Alessandra Bortoluzzi ${ }^{4}$, Antonis Fanouriakis ${ }^{2}$, Emmanouil Papastefanakis ${ }^{1}$, Eleni Kalogiannaki ${ }^{1}$, Irini Gergianaki ${ }^{1}$, Prodromos Sidiropoulos ${ }^{1}$, Dimitrios Boumpas ${ }^{2}$, George Bertsias ${ }^{1} .{ }^{1}$ Rheumatology, Clinical Immunology and Allergy, University of Crete, Heraklion, Greece; ${ }^{2}$ Rheumatology Clinic, 'Attikon' University Hospital and BRFAA, Athens, Greece; ${ }^{3}$ Institute of Computer Science, Foundation for Research and Technology Hellas (FORTH), Heraklion, Greece; ${ }^{4}$ Section of Rheumatology, University of Ferrara, Cona (FE), Italy

Background: A joined EULAR/ACR initiative has proposed a new set of classification criteria for SLE based on weighted items and the use of ANA as an entry criterion.

Objectives: To compare the diagnostic performance of the old and new classification criteria against physician diagnosis in an early SLE cohort and examine phenotypic and prognostic differences among patients who are classified with the criteria.

Methods: Adult patients diagnosed by experienced physicians with SLE $(n=690)$ or control diseases $(n=301)$ during 2005-2016 and followed-up at least 6 months. All sets of criteria (ACR 1997, SLICC 2012, EULAR/ACR 2018) were applied. Hazard models were used to calculate elapsed time between the earliest item (of any criterion) and classification. Severity of SLE (BILAG-2004 glossary) and the SLICC/ACR organ damage index were determined.

Results: The SLICC and EULAR/ACR had increased overall sensitivity as compared to the ACR criteria (91.3\%, 88.6\%, 85.7\%, respectively; $\mathrm{p}<0.01)$, especially in early-onset (<3 years) disease $(91.4 \%, 87.3 \%$, $79.9 \%$, respectively; $p<0.001$ ), with comparable specificity rates (ranging 91.0-92.7\%). By combining the three criteria sets, $97.1 \%$ of patients were classified. Both the EULAR/ACR and the SLICC enabled earlier SLE classification (median time-to-classification: 7.1 and 7.6 months, respectively) than the ACR criteria (median 9.1 months). Nevertheless, disease classification was delayed by $>3$ months in $17.3-19.9 \%$ of cases, particularly in neurological SLE (20.0-26.8\%). Comparative analysis of patients who were missed by the criteria revealed significant differences in rates of individual clinical and serological features (Table 1) suggesting that existing criteria may classify non-overlapping groups of patients. Importantly, unclassified patients presented with a high prevalence of moderate/ severe disease (43.3-60\%) and organ damage (30-50\%).

Conclusion: Despite improved sensitivity and earlier classification with the EULAR/ACR and the SLICC criteria, still SLE diagnosis may be missed or delayed even in patients with moderate to severe disease, especially neurological-dominant disease. Simultaneous application in clinical practice assures the greatest capture of patients.
Table 1. Prevalence of clinical and immunological features across groups of SLE patients who were not classified by the criteria

\begin{tabular}{|l|c|c|c|c|c}
\hline \multicolumn{2}{|c|}{} & \multicolumn{3}{|c|}{ Missed by the } & \multicolumn{1}{l}{} \\
\hline & $\mathbf{A C R ~ 1 9 9 7}$ & $\begin{array}{c}\text { SLICC } \\
\mathbf{2 0 1 2}\end{array}$ & $\begin{array}{c}\text { EULAR/A } \\
\text { CR 2018 }\end{array}$ & $\begin{array}{c}\text { All three } \\
\text { criteria }\end{array}$ & \\
\hline SLICC 2012 items & $\mathbf{n = 9 9}$ & $\mathbf{n = 6 0}$ & $\mathbf{n = 7 9}$ & $\mathbf{n = 2 0}$ & \\
\hline ACLE & $49.5 \%$ & $80.0 \%$ & $72.2 \%$ & $60.0 \%$ & $<0.001$ \\
\hline CCLE & $10.1 \%$ & $8.3 \%$ & $17.7 \%$ & $5.0 \%$ & 0.206 \\
\hline Alopecia & $42.4 \%$ & $36.7 \%$ & $63.3 \%$ & $45.0 \%$ & 0.008 \\
\hline Ulcers & $12.1 \%$ & $23.3 \%$ & $51.9 \%$ & $15.0 \%$ & $<0.001$ \\
\hline Synovitis & $69.7 \%$ & $86.7 \%$ & $74.7 \%$ & $65.0 \%$ & 0.076 \\
\hline Serositis & $6.1 \%$ & $10.0 \%$ & $12.7 \%$ & $10.0 \%$ & 0.506 \\
\hline Renal disorder & $3.0 \%$ & $3.3 \%$ & $6.3 \%$ & $10.0 \%$ & 0.455 \\
\hline Neurologic disorder & $8.1 \%$ & $6.7 \%$ & $6.3 \%$ & $20.0 \%$ & 0.232 \\
\hline Hemolytic anemia & $3.0 \%$ & $0.0 \%$ & $2.5 \%$ & $0.0 \%$ & 0.503 \\
\hline Leukopenia & $20.2 \%$ & $15.0 \%$ & $27.8 \%$ & $10.0 \%$ & 0.166 \\
\hline Thrombocytopenia & $18.2 \%$ & $6.7 \%$ & $12.7 \%$ & $10.0 \%$ & 0.205 \\
\hline Anti-DNA & $18.2 \%$ & $3.3 \%$ & $0.0 \%$ & $0.0 \%$ & $<0.001$ \\
\hline Anti-Sm & $2.0 \%$ & $0.0 \%$ & $1.3 \%$ & $0.0 \%$ & 0.664 \\
\hline Anti-phospholipid Ab & $18.2 \%$ & $1.7 \%$ & $11.4 \%$ & $5.0 \%$ & 0.011 \\
\hline Low complement & $43.4 \%$ & $3.3 \%$ & $21.5 \%$ & $5.0 \%$ & $<0.001$ \\
\hline Coombs test & $5.1 \%$ & $0.0 \%$ & $2.5 \%$ & $0.0 \%$ & 0.232 \\
\hline ANA & $84.8 \%$ & $56.7 \%$ & $44.3 \%$ & $50.0 \%$ & $<0.001$ \\
\hline
\end{tabular}

Acknowledgement: This study received funding by the Hellenic Society of Rheumatology \& Professionals Union of Rheumatologists of Greece (protocol number 644).

Disclosure of Interests: Christina Adamichou: None declared, Dionisis Nikolopoulos: None declared, Irini Genitsaridi: None declared, Alessandra Bortoluzzi: None declared, Antonis Fanouriakis Paid instructor for: Amgen, GSK, Speakers bureau: Abbvie, Enorasis, Genesis Pharma, Emmanouil Papastefanakis: None declared, Eleni Kalogiannaki: None declared, Irin Gergianaki: None declared, Prodromos Sidiropoulos: None declared, Dimitrios Boumpas: None declared, George Bertsias: None declared DOI: 10.1136/annrheumdis-2019-eular.6136

\section{FRI0226 \\ LOW EXPRESSION OF ESTROGEN RECEPTOR BETA IN RENAL TUBULAR EPITHELIAL CELL MAY CONTRIBUTE TO HYPERURICEMIA IN PREMENOPAUNITED STATES OF AMERICAL FEMALE SYSTEMIC LUPUS ERYTHEMATOSUS PATIENTS}

Haijun $\mathrm{Liu}^{1,2}$, Xiaoyan $\mathrm{Cai}^{3,4}$, Lie Dai ${ }^{5}$, MA Jianda ${ }^{5}$, Yingqian Mo ${ }^{5}$, Minyan Xie ${ }^{1}$.

${ }^{1}$ Panyu Central Hospital, Department of Rheumatology, Guangzhou, China; ${ }^{2}$ Jinan University, Guangzhou, China; ${ }^{3}$ Guangzhou First People's Hospital, Department of Rheumatology, Guangzhou, China; ${ }^{4}$ The Second Affiliated Hospital of South China University of Technology, Guangzhou, China; ${ }^{5}$ Sun Yat-Sen Memorial Hospital, Sun Yat-Sen University, Department of Rheumatology, Guangzhou, China

Background: We have reported that the incidence of hyperuricemia of young female systemic lupus erythematosus (SLE) patients was higher than that of healthy young women ${ }^{[1]}$. Whether the estrogen receptor (ER) expressed in renal tubular tissue contributes to it? There are few reports yet.

Objectives: To investigate the expression of ERs in the renal tubular cells and their relationship with serum uric acid (UA) levels in premenopausal female SLE patients.

Methods: Eighteen kidney biopsy specimens of premenopausal female SLE patients served as the lupus nephritis group (LN group), and 12 specimens of premenopausal female $\lg A$ nephropaty patients served as the IgA group. Serum UA levels and kidney index were collected, and the expression of ERs in the renal tubular epithelial cell of the two groups were determined by immunohistochemistry. According to the 